\title{
IL-6 mediates hypoferremia of inflammation by inducing the synthesis of the iron regulatory hormone hepcidin
}

\author{
Elizabeta Nemeth, ${ }^{1}$ Seth Rivera, ${ }^{1}$ Victoria Gabayan, ${ }^{1}$ Charlotte Keller, ${ }^{2}$ Sarah Taudorf, ${ }^{2}$ \\ Bente K. Pedersen, ${ }^{2}$ and Tomas Ganz ${ }^{1}$
}

\begin{abstract}
1Department of Medicine, David Geffen School of Medicine, University of California, Los Angeles (UCLA), Los Angeles, California, USA. ${ }^{2 T h e}$ Copenhagen Muscle Research Centre and The Department of Infectious Diseases, Rigshospitalet, Copenhagen, Denmark.
\end{abstract}

\begin{abstract}
Hypoferremia is a common response to systemic infections or generalized inflammatory disorders. In mouse models, the development of hypoferremia during inflammation requires hepcidin, an iron regulatory peptide hormone produced in the liver, but the inflammatory signals that regulate hepcidin are largely unknown. Our studies in human liver cell cultures, mice, and human volunteers indicate that IL-6 is the necessary and sufficient cytokine for the induction of hepcidin during inflammation and that the IL-6-hepcidin axis is responsible for the hypoferremia of inflammation.
\end{abstract}

\section{Introduction}

Hepcidin, a disulfide-rich peptide produced by hepatocytes (1-3), may be the long-anticipated central regulator of iron metabolism. Recent studies indicate that hepcidin (in mice, hepcidin-1) inhibits intestinal iron absorption $(4,5)$, placental iron transport (4), and release of recycled iron from macrophages (5), effectively decreasing the delivery of iron to maturing erythrocytes in the bone marrow. Thus, in mice, ablation of hepcidin expression removed the inhibition and resulted in iron overload resembling human hemochromatosis, with iron excess in most tissues except the macrophage-rich spleen (5). Conversely, in transgenic mice overexpressing hepcidin-1 (4), transplacental and intestinal iron transport was blocked, and the mice died at birth from severe iron deficiency unless given parenteral iron supplementation. Overexpression of the other known murine hepcidin gene, hepcidin-2, had no effect on iron metabolism in mice (6). In accord with the proposed role of hepcidin as a homeostatic regulator of iron transport and erythropoiesis, hepcidin synthesis is regulated by dietary iron and iron stores, as well as by tissue oxygenation. Thus, hepcidin mRNA expression was increased in mice with dietinduced or genetically induced iron overload $(3,7)$ and decreased in mice with anemia caused by bleeding or hemolysis or in mice with hypoxemia due to decreased ambient oxygen (8).

There is a single human hepcidin gene whose essential role in iron homeostasis was confirmed by identifying homozygous frameshift or nonsense mutations in affected individuals with severe juvenile hemochromatosis (9). Hepcidin production was also diminished in another form of juvenile hemochromatosis due to mutations in the hemojuvelin gene (10) and in the most common form of hemochromatosis, that caused by mutations in the HFE gene $(11,12)$. Conversely, autonomous overexpression of hepcidin mRNA expression was seen in large hepatic adenomas

Nonstandard abbreviations used: anemia of inflammation (AI); Iscove's modified Dulbecco's medium (IMDM); medium from LPS-stimulated macrophages (M-LPS); medium from unstimulated macrophages (M); recombinant human IL-6 (rhIL-6); total iron-binding capacity (TIBC); unsaturated iron-binding capacity (UIBC).

Conflict of interest: The authors have declared that no conflict of interest exists.

Citation for this article: J. Clin. Invest. 113:1271-1276 (2004).

doi:10.1172/JCI200420945. associated with iron-refractory anemia (13). These observations suggest that human hepcidin is the functional equivalent of hepcidin-1 in mice.

Structurally, hepcidin is similar to disulfide-rich antimicrobial peptides such as those produced in the fat body of insects (the equivalent of the vertebrate liver) in response to infections $(2,14)$. In agreement with a potential role for hepcidin in host defense, hepcidin mRNA was increased in the livers of LPS-treated mice and in LPS-treated hepatocytes (3). This also suggested that hepcidin could be the pathogenic mediator of anemia of inflammation (AI), also called anemia of chronic disease. AI is a common condition that affects patients with acute and chronic infections, inflammatory disorders, and neoplastic diseases $(15,16)$. It is characterized by low serum iron levels (hypoferremia), low serum iron-binding capacity, and normal to elevated ferritin concentrations (17). This decreased availability of iron may be a host defense mechanism against invading microorganisms $(15,18)$. The pathogenesis of AI has been attributed to hypoferremia due to impaired mobilization of iron stores from macrophages and decreased iron absorption $(15,19)$, but iron-independent effects such as shortened red cell survival and direct suppression of erythropoiesis could also contribute. We showed that patients with AI due to inflammatory disorders or infections had markedly increased excretion of urinary hepcidin (12). In vitro stimulation of fresh human hepatocytes with a panel of cytokines showed strong induction of hepcidin mRNA by IL-6, but not IL-1 $\alpha$ or TNF- $\alpha$ (12), indicating that IL- 6 may be the mediator of hepcidin induction by inflammation. Additionally, in a mouse model of inflammation, injections of turpentine increased hepcidin mRNA and decreased serum iron (8). Importantly, the turpentine-induced hypoferremia was not observed in hepcidin-deficient mice (8), demonstrating that hepcidin is required for iron sequestration during inflammatory conditions. We therefore asked whether the effects of inflammation on iron metabolism were mediated by the IL-6-hepcidin axis.

\section{Methods}

Macrophage culture. Monocytes from peripheral blood were isolated by centrifugation through Ficoll-Paque (Amersham Pharmacia Biotech, Piscataway, New Jersey, USA) and $46 \%$ iso- 
osmotic Percoll gradient $(12,20)$ (Sigma-Aldrich, St. Louis, Missouri, USA) and their purity confirmed by Wright's stain. Kupffer cells were isolated from collagenase-digested livers (Liver Tissue Procurement and Distribution System, University of Minnesota, Minneapolis, Minnesota, USA, NIH contract N01-DK-9-2310) by repetitive washing and centrifugation at $19 \mathrm{~g}$ and $820 \mathrm{~g}$, followed by selective adherence to plastic. Immunostaining with anti-human CD68 Ab's (PharMingen, San Diego, California, USA) showed that the Kupffer cell culture was $80 \%$ pure. Monocyte-derived macrophages and Kupffer cells were cultured at $10^{6}$ cells/ml in Iscove's modified Dulbecco's medium (IMDM) supplemented with $10 \%$ human serum. The cells were incubated with $100 \mathrm{ng} / \mathrm{ml}$ LPS (Escherichia coli 055:B5) and the cell-free medium from unstimulated macrophages (M) or from LPS-stimulated macrophages (M-LPS) collected after 24 hours.

IL-6 neutralization and cytokine assays. Human hepatocytes (Liver Tissue Procurement and Distribution System) were cultured in human hepatocyte maintenance medium (Clonetics Corp., San Diego, California, USA) at $37^{\circ} \mathrm{C}, 5 \% \mathrm{CO}_{2}$. Hep3B cells (American Tissue Culture Collection, Manassas, Virginia, USA) were cultured in IMDM with $10 \% \mathrm{FCS}$ at $37^{\circ} \mathrm{C}, 5 \% \mathrm{CO}_{2}$. Primary hepatocytes and Hep3B were treated for 24 hours with $100 \mathrm{ng} / \mathrm{ml} \mathrm{LPS}$, with M-LPS (final concentration $16.6 \%$ ), or with the indicated cytokines (R\&D Systems Inc., Minneapolis, Minnesota, USA). The treatments were performed with or without the addition of IL- 6 neutralizing Ab's or TNF- $\alpha$ neutralizing Ab's ( $6 \mu \mathrm{g} / \mathrm{ml}$; R\&D Systems Inc.).

Animals. Animal studies were performed in conformity with applicable laws and guidelines and were approved by the Animal Research Committee at UCLA. Two pairs of IL-6 ${ }^{-/-}$(IL-6 KO) mice on a C57BL/ 6 background (The Jackson Laboratory, Bar Harbor, Maine, USA) were bred in our rodent facility. WT C57BL/6 mice were either obtained directly from The Jackson Laboratory or from The Jackson Laboratory-derived breeders in our UCLA facility. All mice were maintained on either NIH 31 rodent diet (iron content 336 mg/kg; Harlan Teklad, Indianapolis, Indiana, USA) or Prolab RMH 2000 breeder diet (iron content 440 mg/kg; PMI Nutrition International Inc., Brentwood, Missouri, USA). Based on our pilot studies, hepatic hepcidin mRNA was high and not inducible by LPS, parenteral iron, or turpentine in C57B16 mice maintained on such a high-iron content diet (compared with estimated minimal daily requirement of $35 \mathrm{mg} / \mathrm{kg}$ diet) (21). Therefore, $12-15$ days prior to the experiment, mice were switched to an iron-deficient diet containing 2-4 ppm iron (Harlan Teklad).

Turpentine abscess. Mice were anesthetized with isoflurane (Abbott Laboratories, North Chicago, Illinois, USA), and the interscapular area was shaved and scrubbed with $70 \%$ ethanol. Mice then received a $100-\mu 1$ injection of pure gum spirits of turpentine (Sunnyside Corp., Wheeling, Illinois, USA) or $0.9 \%$ sterile PBS in the interscapular fat pad. Mice were sacrificed 16 hours later.

Mouse tissue collection. Blood was collected by cardiac puncture and stored on ice for at least 30 minutes in polypropylene tubes to induce clotting. Serum was then obtained by two-stage centrifugation. The abdomen was opened, and a small piece of liver (less than $100 \mathrm{mg}$ ) was immediately homogenized in TRIzol (Invitrogen Corp., Carlsbad, California, USA).

Quantitative RT-PCR. RNA was prepared using TRIzol reagent according to the manufacturer's instructions. The cDNA was synthesized using iScript cDNA Synthesis Kit (Bio-Rad Laboratories Inc., Hercules, California, USA). Human hepcidin and G3PDH, as well as mouse haptoglobin expression, were analyzed using iQ SYBR
Green Supermix (Bio-Rad Laboratories Inc.). Mouse hepcidin-1 and $\beta$-actin expression were analyzed using a multiplex approach with a degradable quenched probe. The primers were as follows: human hepcidin, 5'-CCTGACCAGTGGCTCTGTTT-3' and 5'CACATCCCACACTTTGATCG-3'; human G3PDH, 5'-TGGTATCGTGGAAGGACTC-3' and 5'-AGTAGAGGCAGGGATGATG-3'; mouse hepcidin-1, sense 5'-CCTATCTCCATCAACAGATG-3', antisense $5^{\prime}$-AACAGATACCACACTGGGAA-3', and probe 5'-FAMCCCTGCTTTCTTCCCCGTGCAAAG-Black Hole Quencher-3'; mouse $\beta$-actin, sense $5^{\prime}$-ACCCACACTGTGCCCATCTA-3', antisense $5^{\prime}$-CACGCTCGGTCAGGATCTTC-3', and probe 5'-Texas red-ATGCTCTCCCTCACGCCATCCTGC-Black Hole Quencher3'; mouse haptoglobin, 5'-AACTCCCCGAATGTGAGGCA-3' and 5'-CGTGGCGGGAGATCATCTTG-3'. Amplification was performed at $52-58^{\circ} \mathrm{C}$ for 40 cycles in iCycler Thermal Cycler (Bio-Rad Laboratories Inc.), and data were analyzed using iCycler iQ Optical System Software. The relative expression in each sample was calculated by a mathematical method based on the real-time PCR efficiencies (22) using as references mRNA mouse $\beta$-actin for murine cells and tissues and human G3PDH for human cells. Because of the wide distribution of the relative expressions in mice, medians and nonparametric statistics were used to describe the data.

Human studies. All human studies were performed in accordance with local regulations and the Declaration of Helsinki. Informed consent was obtained from all subjects.

IL-6 infusion in humans. The study was approved by the Ethical Committee of Copenhagen and Frederiksberg Communities, Denmark. Six healthy volunteers were infused with recombinant human IL-6 (rhIL-6) (Sandoz Ltd., Basel, Switzerland) for 3 hours at the rate of $30 \mu \mathrm{g} /$ hour (23). Urine and serum samples were collected prior to infusion, at the end of a 3-hour infusion, and 2 hours after infusion. Urine was also collected 24 hours after the start of the treatment.

Serum IL-6 measurement. We measured serum IL-6 levels in subjects infused with rhIL- 6 using a human IL-6 ELISA assay (BioSource International, Camarillo, California, USA) according to the manufacturer's recommendations.

Iron supplementation in humans. The study was approved by the Human Subjects Protection Committee at UCLA. Six healthy volunteers collected first morning urine for 9 days. In the morning of days 3, 4, and 5, the subjects ingested $65 \mathrm{mg}$ of iron as ferrous sulfate (Nature Made, Mission Hills, California, USA).

Urinary hepcidin assay. Urinary creatinine concentrations were measured by UCLA clinical laboratories. Cationic peptides were extracted from urine using CM Macro-prep (BioRad Laboratories Inc.) (2) eluted with 5\% acetic acid, lyophilized, and resuspended in $0.01 \%$ acetic acid. Hepcidin urinary concentrations were determined by immunodot assay. Urine extracts equivalent to $0.1-0.5$ $\mathrm{mg}$ of creatinine were dotted on Immobilon-P membrane (Millipore Corp., Bedford, Massachusetts, USA), along with a range of synthetic hepcidin standards (0-40 ng). Hepcidin was detected on the blots using rabbit anti-human hepcidin Ab's (12) with goat anti-rabbit HRP as a secondary Ab. Dot blots were developed by the chemiluminescent detection method (SuperSignal West Pico Chemiluminescent Substrate; Pierce Chemical Co., Rockford, Illinois, USA) and quantified with the Chemidoc cooled camera running Quantity One software (BioRad Laboratories Inc.).

Serum iron measurement. Human and mouse serum iron and unsaturated iron-binding capacity (UIBC) were determined using a colorimetric assay (Diagnostic Chemicals Ltd., Oxford, Connecticut, 
A



B

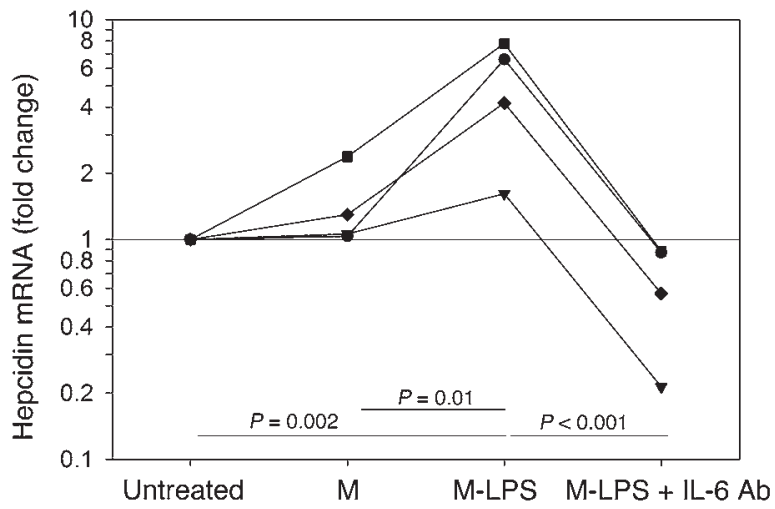

USA), which was modified for the microplate format and verified by testing serial dilutions of the iron standard supplied by the manufacturer. Briefly, $50 \mu \mathrm{l}$ of serum was used for each of the serum iron and UIBC measurements. The total iron-binding capacity (TIBC) was calculated as the sum of serum iron and UIBC, and the percentage of transferrin saturation as serum iron/TIBC $\times 100$.

Statistics. We used Sigma Stat version 3.0 for statistical analyses (Systat Software Inc., Point Richmond, California, USA).

\section{Results}

IL-6 is required for hepcidin induction by microbial stimuli in vitro. We first ascertained whether IL- 6 was a necessary mediator for the induction of hepcidin mRNA by microbial stimuli. LPS treatment increased hepcidin mRNA levels in primary human hepatocytes (Figure 1A), but human hepatoma Hep3B cells were poorly responsive to direct stimulation even by $500 \mathrm{ng} / \mathrm{ml}$ LPS (hepcidin

\section{Figure 2}

The effect of inflammation on hepcidin mRNA and serum iron in WT and IL-6 KO mice. WT (circles) and IL-6 KO (triangles) mice on an iron-depleted diet were treated with a single subcutaneous injection of turpentine. (A) Median expression of hepcidin-1 (normalized to mouse $\beta$-actin) increased 12 -fold in WT mice treated with turpentine $(n=13)$ compared with PBS-treated controls ( $n=10, P<0.001$ by Mann-Whitney rank sum test). Conversely, expression fell 6.4-fold in turpentine-treated IL-6 KO mice $(n=13)$ compared with controls $(n=11, P=0.014) . P<0.001$ for the comparison of WT to IL-6 KO mice (by two-way ANOVA). (B) Mean serum iron fell $49 \%$ in WT mice treated with turpentine $(n=9)$ compared with controls $(n=7, P=0.015$ by Student's $t$ test), whereas there was a slight and marginally significant increase in median serum iron of IL-6 KO mice ( $n=9$ and $n=7$, respectively; $P=0.049$ ). The difference between iron decrease in WT and IL-6 $\mathrm{KO}$ mice was highly significant $(P<0.001$ by two-way ANOVA).

\section{Figure 1}

Anti-IL-6 Ab's neutralize the induction of hepcidin mRNA. Hepcidin mRNA was analyzed relative to G3PDH mRNA by quantitative RT-PCR (qRT-PCR). $P$ values were determined by one-way RMANOVA with a Tukey test. (A) Primary hepatocytes ( $n=4$ donors): untreated (0), treated for 24 hours with $100 \mathrm{ng} / \mathrm{ml}$ LPS (LPS), or treated with $100 \mathrm{ng} / \mathrm{ml} \mathrm{LPS}+6 \mu \mathrm{g} / \mathrm{ml}$ anti-IL-6 Ab's (LPS + IL-6 Ab's). (B) Hep3B cells: untreated (0), treated for 24 hours with $16.6 \%$ medium from monocyte-derived macrophages ( $n=2$ donors) or Kupffer cells ( $n=2$ donors) either unstimulated (M) or stimulated with $100 \mathrm{ng} / \mathrm{ml}$ LPS (M-LPS) or treated for 24 hours with the same $16.6 \% \mathrm{M}-\mathrm{LPS}+6$ $\mu \mathrm{g} / \mathrm{ml}$ anti-IL-6 Ab's (M-LPS + IL-6 Ab's).

mRNA changed 1.16 -fold \pm 0.11 -fold, mean \pm SD, $n=4$ experiments). Hep3B hepcidin mRNA, however, markedly increased after exposure to M-LPS (Figure 1B) or after 24-hour stimulation with $100 \mathrm{ng} / \mathrm{ml}$ IL-6 (4.9-fold \pm 1 .4-fold, mean $\pm \mathrm{SD}, n=5$ experiments). Primary hepatocytes were previously shown to produce IL-6 in response to LPS stimulation (24), raising the possibility that direct LPS stimulation, as well as indirect, LPS-macrophagemediated induction of hepcidin depended on the intermediate production of IL-6. Indeed, both the effects of LPS on primary human hepatocytes and the effects of M-LPS on Hep3B cells were completely reversed when IL-6-neutralizing Ab's were added (Figure 1). Anti-TNF- $\alpha$ Ab's had no detectable effect in either experiment (data not shown). Anti-IL-6 Ab's had similar inhibitory effects when hepatocytes or Hep3B cells were treated with conditioned medium from macrophages exposed to peptidoglycan from Staphylococcus aureus (data not shown).
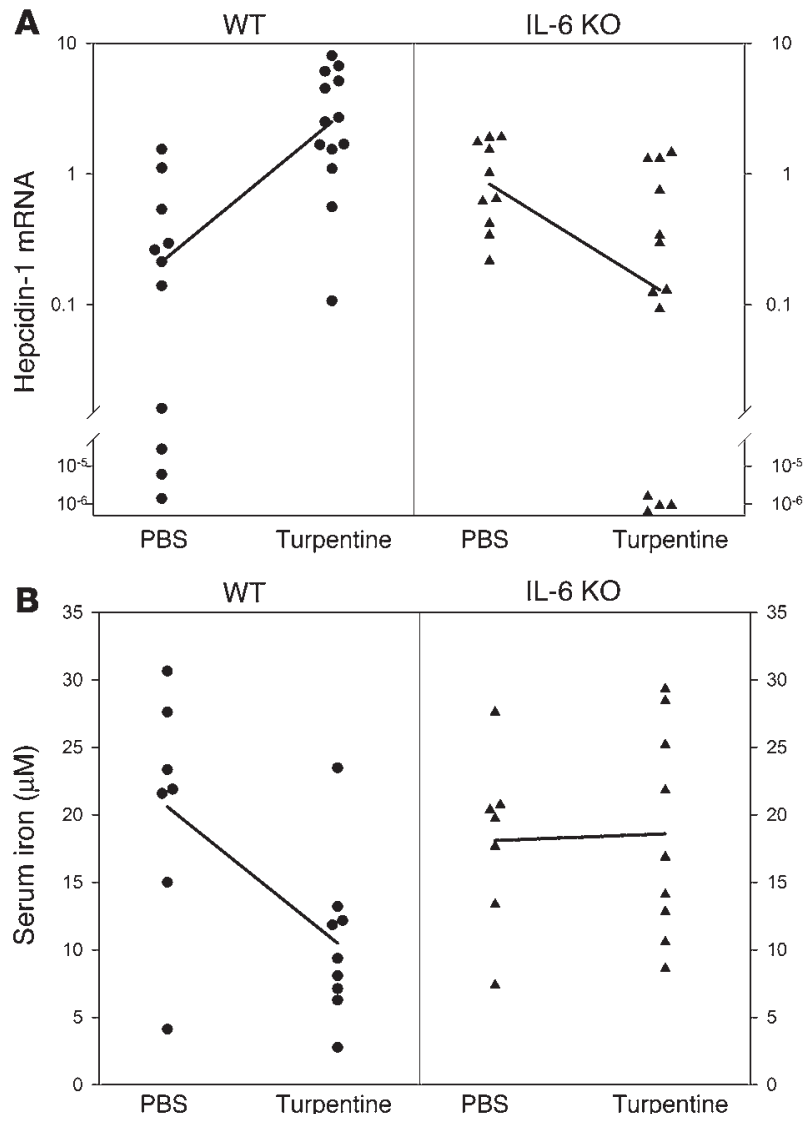


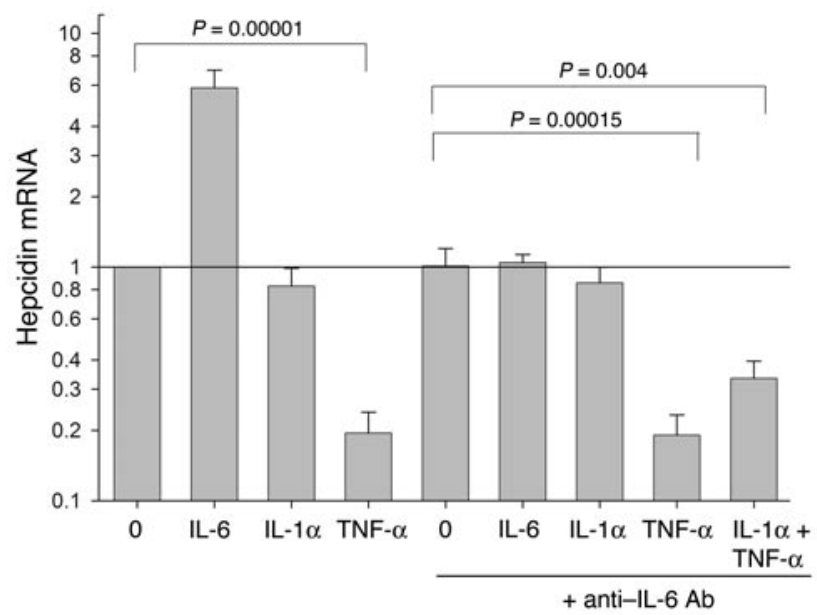

Figure 3

Counterregulation of hepcidin mRNA expression by TNF- $\alpha$. Human hepatoma Hep3B cells were treated with $20 \mathrm{ng} / \mathrm{ml}$ of the indicated cytokine(s) with or without anti-IL-6 Ab's for 24 hours. Their hepcidin mRNA was then analyzed relative to G3PDH mRNA by qRT-PCR. In each experiment, the hepcidin mRNA was expressed as a ratio to that of untreated cells (0), and the mean and standard deviation of four experiments are shown. Paired Student's $t$ test was used to determine the significance of the effects of TNF- $\alpha$.

IL-6 is required for hepcidin induction and bypoferremia during inflammation in mice. To determine whether IL-6 was necessary for the induction of hepcidin by inflammatory stimuli in vivo we created turpentine abscesses in IL-6 KO $(n=13)$ and strain-matched WT mice $(n=13)$. The respective controls were injected with PBS $(n=10$ for IL- $6 \mathrm{KO}$ and $n=11$ for WT). Median hepcidin- 1 expression in the liver increased 12-fold in WT mice treated with turpentine compared with treatment with PBS, but decreased below baseline in turpentine-treated IL-6 KO mice (Figure 2A). Serum iron levels were 49\% lower in turpentine-treated WT mice than in PBS-treated mice. In contrast, median serum iron was slightly higher in turpentine-treated IL-6 KO mice compared with PBS-treated mice (Figure 2B). To ensure that turpentine inflammation generated IL-6, we measured the hepatic expression of haptoglobin, an IL-6-regulated gene (25). Compared with PBS-treated controls, median haptoglobin mRNA was 9.3-fold higher in turpentine-treated WT mice but only 1.9-fold higher in IL-6 KO mice $(P<0.001$ by two-way ANOVA).

Counterregulation of hepcidin by TNF- $\alpha$. The inhibition of hepcidin mRNA expression in stimulated hepatocytes to below baseline by anti-IL-6 Ab's (Figure 1) and the paradoxical decrease of hepcidin

\section{Figure 4}

IL-6 infusion increases urinary hepcidin and decreases serum iron in humans. Six subjects were infused with rhIL-6 for 3 hours at the rate of $30 \mu \mathrm{g} / \mathrm{h}$. Urine and serum samples were collected: prior to infusion at 0 hours, at the end of the infusion ( $3 \mathrm{~h}$ inf), 2 hours after the infusion ( $2 \mathrm{~h}$ after), and 24 hours after the infusion ( $24 \mathrm{~h}$ ). (A) Serum IL-6 concentrations. (B) Urinary hepcidin levels. Thick line represents the geometric mean. The increase in hepcidin at 2 hours after infusion is highly significant $(P<0.001$ by one-way RM ANOVA). (C) Serum iron concentration. (D) Serum transferrin saturation. Thick lines represent the arithmetic mean. The decreases in serum iron concentration and transferrin saturation at 2 hours after infusion are both highly significant compared with preinfusion values (both $P<0.001$ by one-way RM ANOVA). creat, creatinine.
mRNA in turpentine-treated IL-6 KO mice (Figure 2A) raised the possibility that some acute-phase cytokines suppress hepcidin mRNA expression. Indeed, in the Hep3B human hepatoma cell line, TNF- $\alpha$ markedly suppressed hepcidin mRNA levels by a mechanism that did not involve IL- 6 regulation (Figure 3 ). IL-1 $\alpha$ did not have a significant effect in this system.

IL-6 increases hepcidin and induces hypoferremia in humans. Six subjects received IL- 6 by infusion for 3 hours, at which point their serum IL-6 levels reached maximum (Figure 4A), followed by a return to the baseline levels during the next 2 hours. IL- 6 infusion rapidly increased hepcidin excretion (Figure 4B); within 2 hours after the infusion, urinary hepcidin levels were 7.5 -fold higher on average than at 0 hours. After 24 hours, hepcidin concentrations declined toward baseline levels. Importantly, IL-6 infusion also caused a decrease in serum iron and transferrin saturation (Figure 4, C and D). Two hours after the infusion, when hepcidin excretion was at its highest, serum iron decreased on average $34 \%$ and transferrin saturation decreased $33 \%$ in comparison to preinfusion values.

Hepcidin response to dietary iron. Hepatic hepcidin mRNA levels in mice were shown to increase in response to increased dietary iron $(3,26)$, but the analogous human response was not known. Five subjects took $65 \mathrm{mg}$ of iron (as ferrous sulfate) for 3 days, and their urinary hepcidin was monitored before, during, and after iron supplementation. Within 24 hours after the first dose of iron, hepcidin excretion increased 5.4-fold on average (Figure 5). By the next day, however, hepcidin excretion returned to the baseline levels, even though the subjects continued taking iron supplements for 2 more days. We then used IL- $6 \mathrm{KO}$ mice to determine whether iron-induced acute hepcidin increase requires IL-6. Mice that had received an iron-deficient diet (2-4 $\mathrm{mg}$ iron $/ \mathrm{kg}$ diet) for 2 weeks were placed on a standard diet (336 mg iron $/ \mathrm{kg} \mathrm{diet}$ ) for 24 hours. As shown in Figure 6, in WT mice median hepcidin mRNA increased 10.7-fold in iron-refed mice compared with those on an iron-deficient diet, and in IL-6 KO mice hepcidin mRNA increased ninefold. There was no significant difference between WT and KO mice in the hepcidin response to iron refeeding.

\section{Discussion}

We previously showed in human hepatocyte culture (12) that hepcidin is induced by IL- 6 , but not IL- 1 or TNF- $\alpha$, indicating
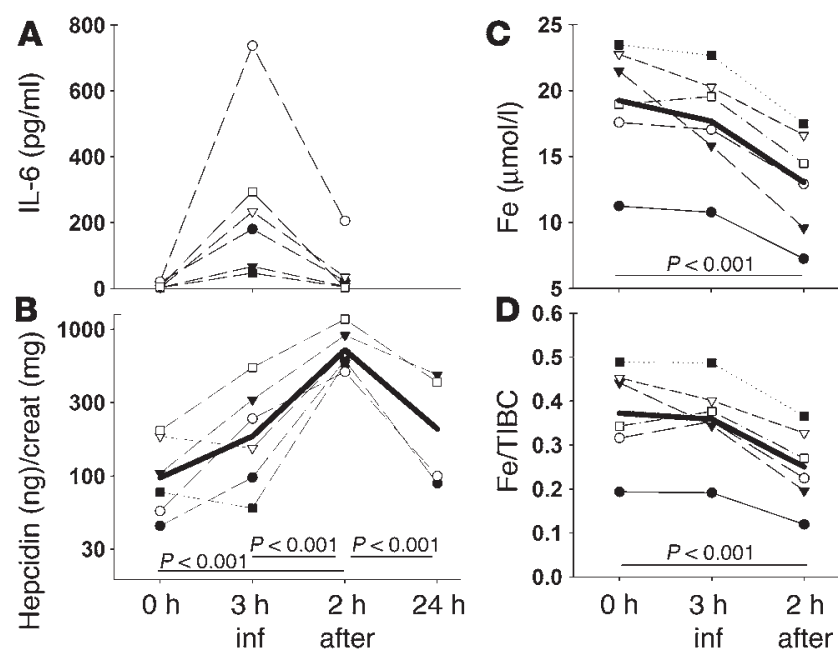


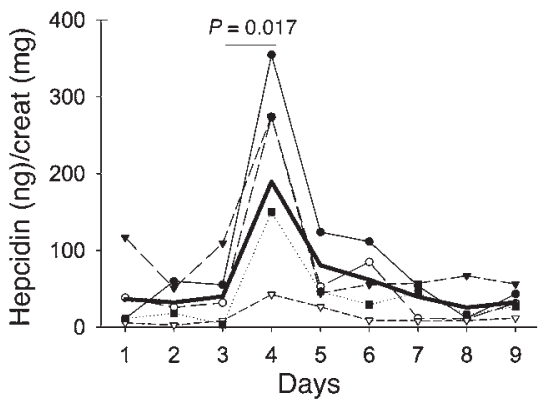

that hepcidin induction by inflammation is a type II acute-phase response. In this study, we demonstrate that IL- 6 is required for the induction of hepcidin and hypoferremia during inflammation and that this cytokine by itself rapidly induces hypoferremia in humans.

In vitro, the addition of IL-6-neutralizing Ab's to hepatocyte culture completely ablated the hepcidin increase induced by LPS or LPS-stimulated macrophages. Thus, from among the many cytokines released from macrophages exposed to LPS (27), IL-6 is the main mediator of hepcidin increase in inflammation. To examine whether the IL-6-hepcidin axis is necessary for the development of hypoferremia in vivo we used the turpentine-induced mouse model of inflammation to compare the response of WT and IL- $6 \mathrm{KO}$ mice. As shown by Nicolas et al. (8), inflammatory hypoferremia in WT mice is mediated by hepcidin because in hepcidin-deficient mice turpentine treatment failed to decrease serum iron. As expected, turpentine significantly induced hepcidin mRNA in WT mice. In IL-6 KO mice, however, hepcidin mRNA was suppressed, likely due to the negative regulation of hepcidin synthesis by other acute-phase cytokines. This interpretation is supported by the suppressive effect of TNF- $\alpha$ on hepcidin mRNA expression in Hep3B cells. The potentially complex interactions of acute-phase cytokines could affect the timing, intensity, and duration of hepcidin response to inflammatory stimuli. Importantly, changes in serum iron concentrations reflected the pattern of hepcidin induction: turpentine treatment caused serum iron decrease in WT mice, but no change in iron levels was detected in IL-6 KO mice. The mechanism by which hepcidin causes hypoferremia remains unclear. Even though the biological effects of hepcidin $(4,5,28)$ strongly suggest it directly inhibits iron transport and recycling, the specific molecular pathways by which this is accomplished remain to be delineated.

In contrast to the requirement for IL- 6 for induction of hepcidin during inflammation, regulation of hepcidin mRNA by iron does not require IL- 6 since iron refeeding increased hepcidin mRNA expression in IL-6 KO mice nearly as much as in WT mice. Thus, there are at least three major and distinct pathways of regulation of hepcidin: regulation by inflammation, dependent on IL-6; regulation by iron, dependent on some other factor, perhaps transferrin saturation; and suppression of hepcidin production during hypoxia and anemia (8) (and our unpublished data). The inflammatory and iron pathways of hepcidin stimulation respond rapidly, with major hepcidin increase detectable within 1 day in both humans and mice. It is remarkable that in humans the stimulatory effect of dietary iron on hepcidin is largely lost after the first dose of iron. We surmise that the second and third iron doses present a weaker stimulus to hepcidin production because iron absorption is greatly diminished by the "mucosal block" phenomenon (29-31), and the rise in transferrin saturation is much smaller

\section{Figure 5}

Iron ingestion induces urinary hepcidin in humans. Five subjects collected their first morning urine for 9 days and took $65 \mathrm{mg}$ of iron (as ferrous sulfate) in the morning of days 3,4 , and 5 . Thick line represents the arithmetic mean. There is a significant increase in hepcidin excretion on day 4 ( 24 hours after the first dose of iron) compared with day $3(P=0.017$ by paired Student's $t$ test).

from the second and third dose than from the first one (32). It remains to be seen whether the mucosal block to iron absorption is due to the inhibitory effects of hepcidin on iron transport by enterocytes or whether the enterocytes limit further iron uptake in response to their own increased cellular iron content.

Our human studies of the effects of IL- 6 parallel the findings in cell cultures and the mouse model. Infusion of IL- 6 rapidly (within hours) increased hepcidin excretion. Because of the high rate of blood filtration in kidneys (the entire plasma volume is filtered through the kidneys in less than an hour), the observed urinary hepcidin levels should closely reflect the timeline of its production. Concomitant with hepcidin increase after IL-6 infusion, we observed a significant decrease in serum iron and transferrin saturation. The rapid IL-6-induced decrease in transferrin saturation argues strongly against hemodilution (33) as the cause of IL-6-mediated hypoferremia. In a model that we proposed (12, $34)$ as an extension of earlier hypotheses $(13,35)$, IL-6-stimulated increase in plasma hepcidin concentration blocks the release of iron from macrophages that recycle iron from senescent erythrocytes. Transferrin-bound iron in plasma (approximately 2-4 mg in

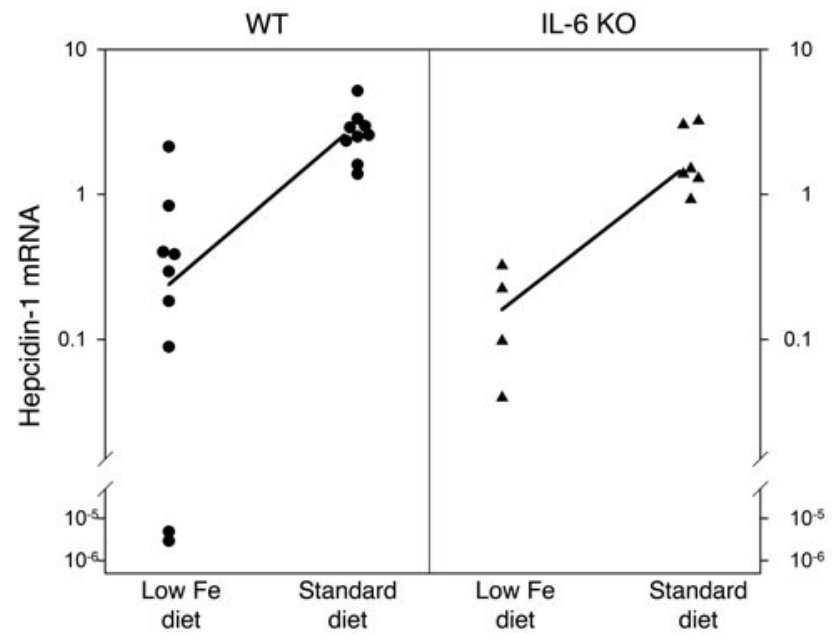

\section{Figure 6}

Iron ingestion induces hepcidin mRNA in WT and IL-6 KO mice. WT (circles) and IL-6 KO (triangles) mice on an iron-depleted diet were switched to a standard diet for 24 hours. Median hepcidin-1 mRNA expression (normalized to mouse $\beta$-actin mRNA) increased significantly in both WT (10.7-fold, $P<0.001$ by Mann-Whitney rank sum test) and IL- $6 \mathrm{KO}$ (ninefold, $P=0.009$ by Student's $t$ test) mice. After the switch to a standard diet, there was no significant difference in hepcidin- 1 mRNA levels in WT and IL- 6 KO mice ( $P=0.536$ by twoway ANOVA on ranks). 
adult humans) represents only about $0.1 \%$ of the total body iron content and functions as a transit compartment that turns over every 4 hours (16). When the rate of iron release from macrophages drops, plasma iron concentrations rapidly decrease. Since the developing erythrocytes constitute the main iron-consuming tissue, the decrease in iron supply becomes a bottleneck for hemoglobin synthesis and eventually results in anemia. In agreement with this model, transgenic overexpression of IL-6 in mice (36) and repeated administration of IL- 6 in rats and cancer patients (33) has been reported to cause anemia. If, indeed, chronic stimulation of the IL-6-hepcidin axis by inflammation, aging-associated cytokine dysregulation (37), or neoplasm (38) is responsible for iron-restricted erythropoiesis commonly seen in these conditions, interventions targeting this pathway could be useful for therapy of the resulting anemia.

1. Krause, A., et al. 2000. LEAP-1, a novel highly disulfide-bonded human peptide, exhibits antimicrobial activity. FEBS Lett. 480:147-150.

2. Park, C.H., Valore, E.V., Waring, A.J., and Ganz, T. 2001. Hepcidin, a urinary antimicrobial peptide synthesized in the liver. J. Biol. Chem. 276:7806-7810.

3. Pigeon, C., et al. 2001. A new mouse liver-specific gene, encoding a protein homologous to human antimicrobial peptide hepcidin, is overexpressed during iron overload. J. Biol. Chem. 276:7811-7819.

4. Nicolas, G., et al. 2002. Severe iron deficiency anemia in transgenic mice expressing liver hepcidin. Proc. Natl. Acad. Sci. U. S. A. 99:4596-4601.

5. Nicolas, G., et al. 2001. Lack of hepcidin gene expression and severe tissue iron overload in upstream stimulatory factor 2 (USF2) knockout mice. Proc. Natl. Acad. Sci. U. S. A. 98:8780-8785.

6. Lou, D.Q., et al. 2004. Functional differences between hepcidin-1 and -2 in transgenic mice. Blood. 103:2816-2821.

7. Ilyin, G., et al. 2003. Comparative analysis of mouse hepcidin 1 and 2 genes: evidence for different patterns of expression and co-inducibility during iron overload. FEBS Lett. 542:22-26.

8. Nicolas, G., et al. 2002. The gene encoding the iron regulatory peptide hepcidin is regulated by anemia, hypoxia, and inflammation. J. Clin. Invest. 110:1037-1044. doi:10.1172/JCI200215686.

9. Roetto, A., et al. 2003. Mutant antimicrobial peptide hepcidin is associated with severe juvenile hemochromatosis. Nat. Genet. 33:21-22.

10. Papanikolaou, G., et al. 2004. Mutations in HFE2 cause iron overload in chromosome $1 \mathrm{q}$-linked juvenile hemochromatosis. Nat. Genet. 36:77-82.

11. Bridle, K.R., et al. 2003. Disrupted hepcidin regulation in HFE-associated haemochromatosis and the liver as a regulator of body iron homoeostasis. Lancet. 361:669-673.

12. Nemeth, E., et al. 2003. Hepcidin, a putative mediator of anemia of inflammation, is a type II acutephase protein. Blood. 101:2461-2463.

13. Weinstein, D.A., et al. 2002. Inappropriate expression of hepcidin is associated with iron refractory anemia: implications for the anemia of chronic disease. Blood. 100:3776-3781.

14. Hunter, H.N., Fulton, D.B., Ganz, T., and Vogel, H.J.

\section{Acknowledgments}

Miguel Lopez, Lan Lin, and Ole Sorensen generously contributed time and reagents to this effort. Niels Borregaard suggested the collaboration between the Danish and US groups.

Received for publication December 30, 2003, and accepted in revised form January 28, 2004.

Address correspondence to: Tomas Ganz, Center for the Health Sciences 37-055, Department of Medicine, David Geffen School of Medicine at UCLA, 10833 Le Conte Avenue, Los Angeles, California 90095, USA. Phone: (310) 825-6112; Fax: (310) 206-8766; E-mail: tganz@mednet.ucla.edu.

Elizabeta Nemeth and Seth Rivera contributed equally to this work.
2002. The solution structure of human hepcidin, a peptide hormone with antimicrobial activity that is involved in iron uptake and hereditary hemochromatosis. J. Biol. Chem. 277:37597-37603.

15. Jurado, R.L. 1997. Iron, infections, and anemia of inflammation. Clin. Infect. Dis. 25:888-895.

16. Andrews, N.C. 1999. Disorders of iron metabolism. N. Engl.J. Med. 341:1986-1995.

17. Erslev, A.J. 2000. Anemia of chronic disease. In Williams Hematology. E. Beutler, M.A. Lichtman, B.S. Coller, T.J. Kipps, and U. Seligsohn, editors. McGraw-Hill Professional. New York, New York, USA. 481-488.

18. Schaible, U.E., Collins, H.L., Priem, F., and Kaufmann, S.H. 2002. Correction of the iron overload defect in beta-2-microglobulin knockout mice by lactoferrin abolishes their increased susceptibility to tuberculosis. J. Exp. Med. 196:1507-1513.

19. Means, R.T., Jr. 1999. Advances in the anemia of chronic disease. Int. J. Hematol. 70:7-12.

20. Liu, L., Roberts, A.A., and Ganz, T. 2003. By IL-1 signaling, monocyte-derived cells dramatically enhance the epidermal antimicrobial response to lipopolysaccharide. J. Immunol. 170:575-580.

21. National Research Council (U.S.) and Subcommittee on Laboratory Animal Nutrition. 1995. Nutrient requirements of laboratory animals. National Academy of Sciences, Washington, D.C. 192 pp.

22. Pfaffl, M.W. 2001. A new mathematical model for relative quantification in real-time RT-PCR. Nucleic Acids Res. 29:e45.

23. Steensberg, A., Fischer, C.P., Keller, C., Moller, K., and Pedersen, B.K. 2003. IL-6 enhances plasma IL-1ra, IL-10, and cortisol in humans. Am. J. Physiol. Endocrinol. Metab. 285:E433-E437.

24. Panesar, N., Tolman, K., and Mazuski, J.E. 1999. Endotoxin stimulates hepatocyte interleukin-6 production. J. Surg. Res. 85:251-258.

25. Ramadori, G., and Christ, B. 1999. Cytokines and the hepatic acute-phase response. Semin. Liver Dis. 19:141-155.

26. Anderson, G.J., et al. 2002. Relationship between intestinal iron-transporter expression, hepatic hepcidin levels and the control of iron absorption. Biochem. Soc. Trans. 30:724-726.

27. Van Amersfoort, E.S., Van Berkel, T.J., and Kuiper,
J. 2003. Receptors, mediators, and mechanisms involved in bacterial sepsis and septic shock. Clin. Microbiol. Rev. 16:379-414.

28. Nicolas, G., et al. 2003. Constitutive hepcidin expression prevents iron overload in a mouse model of hemochromatosis. Nat. Genet. 34:97-101.

29. Frazer, D.M., et al. 2003. A rapid decrease in the expression of DMT1 and Dcytb but not Ireg1 or hephaestin explains the mucosal block phenomenon of iron absorption. Gut. 52:340-346.

30. Hahn, P.F., Bale, W.F., Ross, J.F., Balfour, W.M., and Whipple, G.H. 1943. Radioactive iron absorption by gastro-intestinal tract: influence of anemia, anoxia, and antecedent feeding distribution in growing dogs. J. Exp. Med. 78:169-188.

31. Stewart, W.B., Yuile, C.L., Claiborne, H.A., Snowman, R.T., and Whipple, G.H. 1950. Radioiron absorption in anemic dogs: fluctuations in the mucosal block and evidence for a gradient of absorption in the gastrointestinal tract. J. Exp. Med. 92:375-382.

32. O'Neil-Cutting, M.A., and Crosby, W.H. 1987. Blocking of iron absorption by a preliminary oral dose of iron. Arch. Intern. Med. 147:489-491.

33. Nieken, J., et al. 1995. Recombinant human interleukin- 6 induces a rapid and reversible anemia in cancer patients. Blood. 86:900-905.

34. Ganz, T. 2003. Hepcidin, a key regulator of iron metabolism and mediator of anemia of inflammation. Blood. 102:783-788.

35. Fleming, R.E., and Sly, W.S. 2001. Hepcidin: a putative iron-regulatory hormone relevant to hereditary hemochromatosis and the anemia of chronic disease. Proc. Natl. Acad. Sci. U. S. A. 98:8160-8162.

36. Katsume, A., et al. 2002. Anti-interleukin 6 (IL-6) receptor antibody suppresses Castleman's disease like symptoms emerged in IL-6 transgenic mice. Cytokine. 20:304-311.

37. Ershler, W.B., and Keller, E.T. 2000. Age-associated increased interleukin- 6 gene expression, late-life diseases, and frailty. Annu. Rev. Med. 51:245-270.

38. Barille, S., Bataille, R., and Amiot, M. 2000. The role of interleukin- 6 and interleukin-6/interleukin-6 receptor-alpha complex in the pathogenesis of multiple myeloma. Eur. Cytokine Netw. 11:546-551. 